\title{
Diffraction from stepped surfaces in thermal equilibrium
}

\author{
N.C. Bartelt, T.L. Einstein and Ellen D. Williams \\ Department of Physics, University of Maryland, College Park, MD 20742-4111, USA
}

Received 29 June 1990; accepted for publication 14 September 1990

\begin{abstract}
We have performed Monte Carlo simulations of the diffraction from simple two-dimensional models of vicinal surfaces in order to aid interpretation of measured diffraction profiles. At low temperature, we find the sharp diffraction features predicted from the analogy of stepped surfaces with two-dimensional incommensurate phases. These sharp features vanish only near the roughening temperature of the low-index surface corresponding to the terraces between steps. If one fits experimental data having sharp diffraction features to models of step disorder which do not include the ordering influence of step wandering, one can scverely overestimate the amount of disorder. We emphasize that long-range correlations in step positions are more important than the local order in step edge structure or step separations for interpreting sharp diffraction features from steps. After much theoretical effort, it has become well-established that asymptotically the height-height correlations for rough surfaces diverge logarithmically (with a prefactor having a universal component at low temperature). We show explicitly how to use diffraction data to access this behavior for stepped surfaces. In the process, we evaluate the accuracy of a popular approximate expression for the diffracted intensity.
\end{abstract}

\section{Introduction}

Steps on surfaces play an important role in many surface processes. To understand these processes the characterization of the configuration of steps is necessary. In particular, one would like to determine from diffraction experiments such quantities as the disorder in step edges and the fluctuations in the distances between steps. Unfortunately, such analysis of distribution functions from measured diffraction profiles is fraught with problems of non-uniqueness. Thus to provide a basis for interpreting experimental results, we discuss the expected diffraction signatures for various physical models which might govern step behavior under experimental conditions. For stepped surfaces below the roughening temperature of the nearby low-index surface, the fundamental source of disorder in thermal equilibrium is step wandering. As we shall illustrate throughout this paper, the equilibrium step behavior cannot be predicted correctly in a one-dimensional description, since step wandering is omitted by definition in one dimension. In this paper we address the problem of characterization of equilibrium step behavior by computing the diffraction from simple model surfaces using the Monte Carlo method.

Diffraction experiments are most sensitive to step disorder at conditions where neighboring terraces scatter out-of-phase [1]. At out-of-phase conditions, straight steps which are arranged in a perfectly ordered staircase will give rise to "split beams", the spacing between the split beams being proportional to the inverse of the step spacing. The introduction of step wandering into the step configuration will broaden these beams. The amount of step disorder consistent with a diffraction pattern with well-defined splittings, i.e., with a well-defined step periodicity, has been the subject of a number of discussions based on purely statistical one-dimensional models [2], without addressing the issuc of the physical mechanisms responsible for the disorder. In devising models of step disorder, it is important to respect the behavior one expects for correlations in the step positions. Step distributions which arise from a one-dimensional statistical mechanical model with a given set of interactions will have much smaller height-height correlations than one expects of a two-dimensional surface described by the same 
interactions, and, as a result, the splittings will be less well-resolved [or absent] in the $1 \mathrm{D}$ case than in the $2 \mathrm{D}$ case. In this paper we present the results of an explicit computation of the scattering from very simple two-dimensional statistical mechanical models of vicinal surfaces, which show that there are well-defined splittings in situations where there is considerable (short-range!) step disorder. The reason for this behavior is that two-dimensional step wandering enhances correlations between the positions of steps due to an effective entropic repulsion [3]. This result, although perhaps surprising in the context of the one-dimensional results, follows naturally from the analogy of vicinal surfaces with incommensurate phases; two-dimensional incommensurate phases can lead to diffraction features which are difficult to distinguish from true Laue $\delta$-functions [4]. Our work is similar in spirit to the Monte Carlo work of Selke and Szpilka for high-Miller-index surfaces near the roughening transition [5]. We differ in that we consider surfaces with larger step separations, and with no direct energetic interactions between steps.

\section{The solid-on-solid and terrace-step-kink models of stepped surfaces}

The models we consider are the standard solidon-solid (SOS) model $[3,6]$ and the terrace-step-

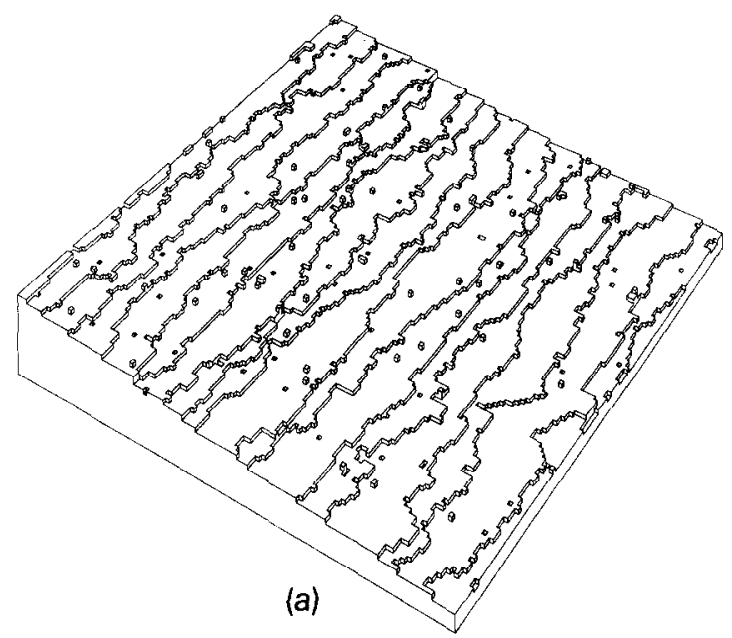

kink (TSK) model [3]. In the SOS model, the height at each site $\boldsymbol{r}_{i}$ of a two-dimensional square grid is described by an integer variable $h\left(\boldsymbol{r}_{i}\right)$. The hamiltonian has the form

$$
I\left(\left\{\boldsymbol{h}\left(\boldsymbol{r}_{i}\right)\right\}\right)=\frac{\epsilon}{2} \sum_{\langle i j\rangle}\left|\boldsymbol{h}\left(\boldsymbol{r}_{i}\right)-\boldsymbol{h}\left(\boldsymbol{r}_{j}\right)\right|,
$$

where the sum is over only nearest neighboring sites and $\epsilon$ is the energy cost of a "broken" nearest-neighbor bond on the surface. In a two-dimensional model, $\epsilon$ is also the energy for forming a "kink" [3] at a step edge. This model includes no energetic interactions between steps, except for implicit hard-core exclusions, and thus is the simplest model of equilibrium step behavior. We have performed Monte Carlo simulations of this model on grids of dimension $L_{x} \times L_{y}$. To obtain a surface with $L_{z}$ steps, we used the following screw boundary conditions:

$h\left(x+L_{x}, y\right)=h(x, y)+L_{z}, \quad$ and

$h\left(x, y+L_{y}\right)=h(x, y)$.

For the most extensive of our computations, we took $L_{x}=L_{y}=128$ and $L_{z}=16$. This corresponds to a mean step separation of $l=L_{x} / 16=8$. If we view our model as vicinal to the $(100)$ face of a cubic crystal, these parameters correspond to an angle of miscut $\phi \approx 7.1^{\circ}$. Fig. 1a shows a sample Monte Carlo configuration of the SOS model vicinal surface at $k_{\mathrm{B}} T=0.7 \epsilon$.

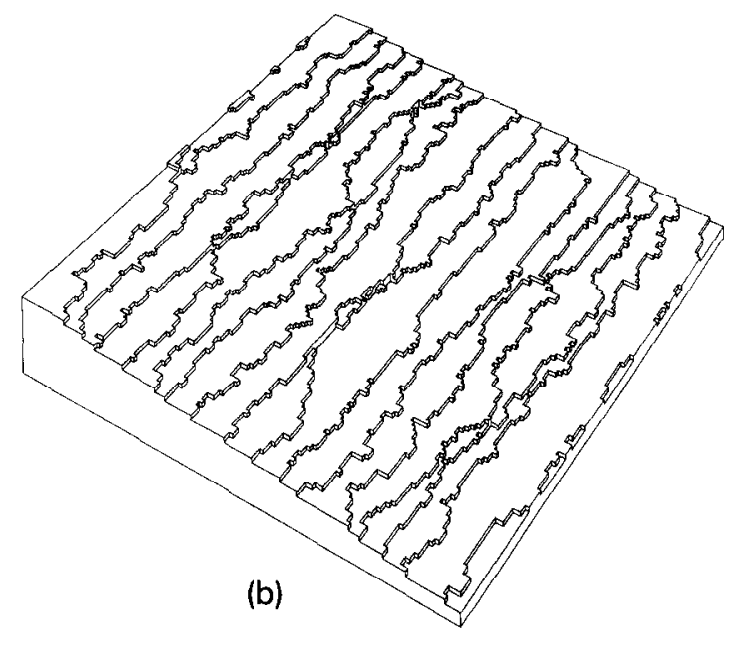

Fig. 1. Sample Monte Carlo configurations of surfaces of dimension $128 \times 128$, with average terrace width of eight lattice constants at $k_{\mathrm{B}} T=0.7 \epsilon$ : (a) the SOS model. (b) the TSK model. 
The terrace-step-kink model is a simplification of the SOS model, in which the terrace excitations are not allowed: the only thermal excitations are "kinks" in the step edges. In the particular model we consider in this paper, each unit length of each step costs energy $\epsilon$. (Thus $\epsilon$ is the kink energy.) At low temperature one expects that the SOS model and this version of the TSK model will yield identical results because the lowest energy excitations of the SOS model arc the excitations of the TSK model. (Terrace excitations of the SOS model cost $4 \epsilon$.) The correspondence between these models is clear in fig. $1 \mathrm{~b}$, which shows a typical Monte Carlo configuration of the TSK model at the same temperature as the SOS configuration in fig. 1a. At high temperatures, however, one does

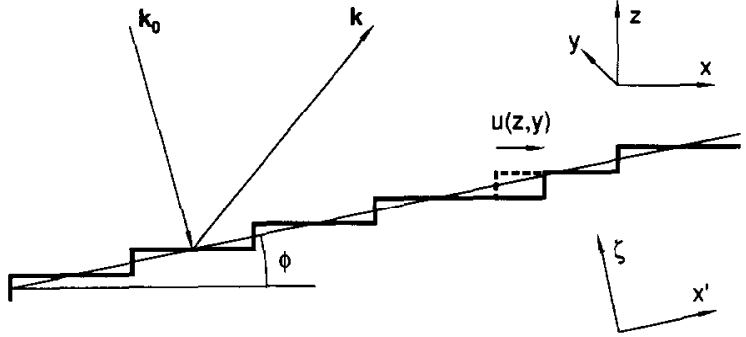

Fig. 2. The coordinate system used in this paper. The displacement of each step in the TSK model from its average position is given by $u(z, y)$.

expect differences, especially above the roughening temperature of the low-index surface of which the terraces are composed. The evolution of these differences will be illustrated below.
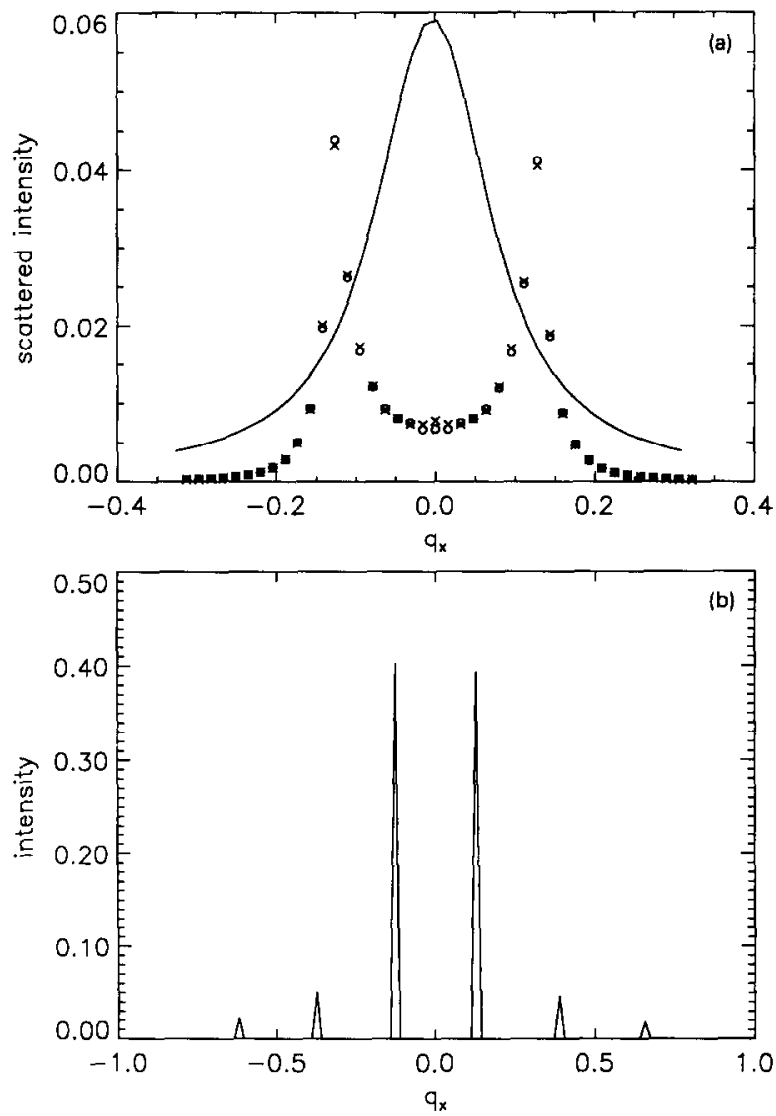

Fig. 3. (a) Computed diffracted profiles at an out-of-phase condition for four models. The solid line is for zero temperature (straight, non-interacting steps). The crosses and circles are for the SOS and TSK model, respectively, at $k_{\mathrm{B}} T=0.7 \epsilon$. At this temperature the two models have the same scattered intensity, to the accuracy of our calculations. (b) Intensity expected for perfectly ordered steps for comparison. $q_{x}$ is in units of $\pi / a$, where $a$ is the lattice constant. 


\section{The role of step wandering on diffraction features}

To mimic diffraction from vicinal surfaces, we compute the scattered intensity in the kinematic approximation as a function of the outgoing momentum $\boldsymbol{k}$ at fixed incident momentum $\boldsymbol{k}_{0}$. In particular, we compute the kinematic structure factor:

$$
S(\boldsymbol{q})=\left\langle\left|\sum_{x, y} \exp \left[\mathrm{i}\left(q_{x} x+q_{y} y+q_{z} h(x, y)\right)\right]\right|^{2}\right\rangle,
$$

where $\boldsymbol{q}=\boldsymbol{k}-\boldsymbol{k}_{0}$ is the momentum transfer in units of reciprocal lattice constants. Our coordinate system is defined in fig. $2: \hat{x}$ and $\hat{y}$ are in the plane of the terraces, with $\hat{\boldsymbol{x}}$ perpendicular to the step edges, and $\hat{z}$ is the terrace surface normal. For concreteness we assume normal incidence to the vicinal surface (i.e., the incident beam is at an angle $\phi$ with respect to the $\hat{z}$-direction as in fig. 2 ), so that $q_{x}=k_{x}-k_{0} \sin \phi, q_{y}=k_{y}$ and $q_{z}=k_{z}+$ $k_{0} \cos \phi$, with $k_{x}^{2}+k_{y}^{2}+k_{z}^{2}=k_{0}^{2}$. Near the specular beam (i.e., $q_{x}=0$ and $q_{y}=0$ ) in-phase conditions $\left(q_{x}=2 \pi n\right)-$ with unsplit diffraction beams - then occur when $k_{0}=n \pi / \cos \phi$; out-of-phase conditions - split diffraction beams - occur when $k_{0}=\left(n+\frac{1}{2}\right) \pi / \cos \phi$.

At zero temperature, no thermal excitations occur, so the ground states of these models are composed of straight steps placed at random positions; the hamiltonian of eq. (1) does not prefer any particular step-step separation. Only in this pathological case are the one- and two-dimensional models equivalent. The degeneracy of the ground state leads to an exponential distribution of step widths. The diffraction from this type of distribution is discussed in ref. [2]. When neighboring planes scatter out-of-phase, the scattered intensity has a Lorentzian shape, as shown by the solid line in fig. $3 \mathrm{a}$ for the out-of-phase condition $k_{0}=3 \pi /(2 \cos \phi)$ : the splitting expected for perfectly ordered steps (fig. 3b) does not occur. This lack of a well-defined splitting is a general result for one-dimensional models with interactions which are much shorter than the average terrace width, $l$. Thus, in a $1 \mathrm{D}$ model, it would be neces- sary to include energetic long-range step-step interactions explicitly to explain experimentally observed splittings. Such energetic interactions are not necessary in a $2 \mathrm{D}$ model.

We computed $S(q)$ for the TSK and SOS models using standard Monte Carlo methods. The starting Monte Carlo configurations usually contained a random distribution of straight steps. We performed several runs at each temperature; in each run we performed $2 \times 10^{5}$ to $2 \times 10^{6}$ Monte Carlo steps per site, accumulating averages every 10 to 40 steps. Typically $\sim 2 \times 10^{4}$ steps were required for equilibration. (Long runs of this length are needed because of the long-range correlations characteristic of vicinal surfaces: see below.) By comparing the results from several different runs, we estimate our computed diffraction profiles are accurate to better than approximately $5 \%$.

The crosses in fig. 3a show the diffracted intensity at an out-of-phase condition, computed for the SOS model at $k_{\mathrm{B}} T=0.7 \epsilon$, the same temperature as the configuration shown in fig. 1a, where there is considerable disorder in the step edges. The diffracted intensity, however, shows a well-defined splitting. Thus, rather remarkably, the step wandering arising from kink formation sharpens the corresponding diffraction pattern. This sharpening is a consequence of the long-range entropic repulsion between steps [7]. This repulsion makes the surface more ordered, in the sense described in the next section, at finite temperature (when step wandering occurs) than at zero temperature, where the behavior is that of a $1 \mathrm{D}$ system. The circles in fig. 3a also show the corresponding diffraction profile for the TSK model (cf. fig. 1b). At this temperature, to the accuracy of our calculation, there is no difference between the TSK and the SOS model.

\section{Approximations to the shape of the diffracted profiles}

In the previous section, we showed that well-resolved beam splittings can be produced by steps wandering thermally, even in the absence of energetic step-step interactions. We now ask what are the quantitative signatures of such freely 
wandering, equilibrated step structures which would appear in measured diffraction profiles. To answer this question, we invoke the statistical mechanical analysis common to models of incommensurate structures and of surface roughening.

The shapes of the diffraction profiles for vicinal surfaces in equilibrium can be estimated from knowledge of the asymptotic form of surface height correlation functions, using the assumptions of Villain et al. [8]. By assuming a Gaussian distribution of the displacements $u(z, y)$ of the steps about their mean positions (see fig. 2), $S(q)$ can be written in terms of the correlations of the displacements (see fig. 2), $g(z, y)=\langle(u(z, y)-$ $\left.u(0,0))^{2}\right\rangle$ :

$$
\begin{aligned}
S(\boldsymbol{q})= & \frac{\left(1-\cos q_{z}\right)}{\left(1-\cos q_{x}\right)} L_{y} L_{z} \sum_{y_{.}} \exp \left\{\mathrm { i } \left[q_{y} y\right.\right. \\
& \left.\left.+\left(q_{z}+q_{x} l\right) z\right]-q_{x}^{2} g(z, y) / 2\right\} .
\end{aligned}
$$

(As written this equation is only valid when $\left|q_{x}\right|$ $\ll 2 \pi$, a condition which is upheld in the calculations near the specular beam presented here.) This equation reveals that for the diffracted intensity what is important near the peaks is not so much local step disorder, but the long-range correlations in the positions of the steps. Whether or not there are sharp diffraction features at an out-of-phase condition will depend on the behavior of $g(z, y)$ at large $z$, not necessarily, for example, on the terrace width distribution.

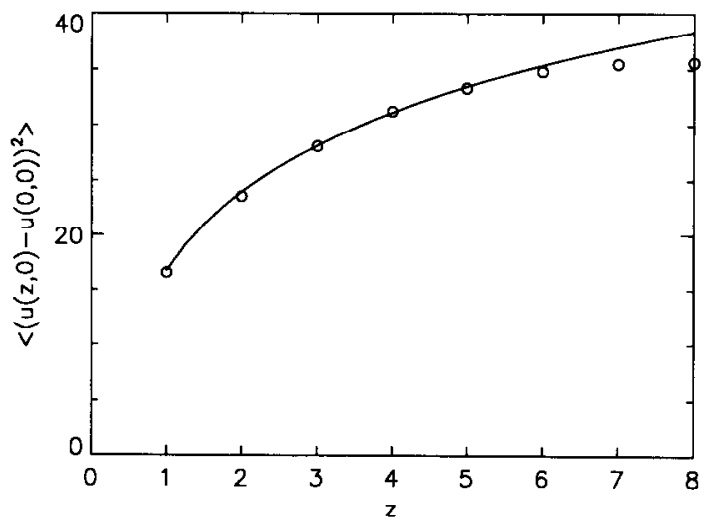

Fig. 4. Comparison between calculated step displacement correlations (circles) and the prediction of eq. (5) for large $z$ with $c_{z}=5.0$ and $X=0.040$ (solid line). $z$ and $u$ are in units of lattice constants.
For one-dimensional models with short-range forces, one expects $g(z, y)$ to diverge linearly with $z$ (in analogy with one-dimensional random walks). This leads (through eq. (4)) to Lorentzianlike peaks, which are generally shifted from their positions when the surface is perfectly ordered, as illustrated in fig. 3a. For two-dimensional surfaces in thermal equilibrium, $g(z, y)$ is expected to diverge only logarithmically at large $z, y$ [8]:

$$
\begin{aligned}
g(z, y) & =\left\langle(u(z, y)-u(0,0))^{2}\right\rangle \\
& \sim \frac{2 X}{\sin ^{2} \phi} \ln \left(c_{z}^{2} z^{2}+c_{y}^{2} y^{2}\right) .
\end{aligned}
$$

The parameter $X$ is a measure of the rate at which the correlations decay with step separation. $X=0$ corresponds to a stepped surface with long-range step order, i.e., a facet on the equilibrium crystal shape. As shown in fig. 4, a fit of Monte Carlo results for the TSK model at $k_{\mathrm{B}} T=0.8 \epsilon$ to the form of eq. (5) for $y=0$ yields $c_{z}=5.0$ and $X=$ 0.040 . (The failure of the fit at large $z$ is presumably due to finite-size effects: because the periodic boundary conditions $g(z, y)=g(16-z, y)$ which reduces the value of $g$ at large $z$.) The values of $X$, $c_{z}$, and $c_{y}$ in general depend on temperature and angle of misorientation. However, as discussed below, reasonable estimates of their values can be made.

For TSK systems with short-range interactions between steps, $X$ takes on a universal (tcmpcrature-independent) value in the limit of small $\phi$. This number can be computed from results in ref. [9]: the form of the height correlations perpendicular to the surface plane (not the terrace) is:

$$
\left\langle\left(\zeta\left(x^{\prime}, y\right)-\zeta(0,0)\right)^{2}\right\rangle \approx \frac{1}{2 \pi^{2}} \ln \left(c_{x}^{2} x^{\prime 2}+c_{y}^{2} y^{2}\right),
$$

where $\zeta\left(x^{\prime}, y\right)$ is the height of the vicinal surface, above $\left(x^{\prime}, y\right)$ on the average surface plane (see fig. 2). By assuming that $\left|\zeta\left(x^{\prime}, y\right)\right| \ll\left|x^{\prime}\right|$ (as expected on the basis of eq. (6)), one obtains eq. (5) with $X=X_{v} \equiv 1 / 4 \pi^{2} \approx 0.025 . \quad X_{v}$ is smaller than the number we observe, but of the same order of magnitude.

The valucs of $c_{z}$ and $c_{y}$ are nonuniversal and will depend on the temperature, i.e., on the amount 
of step wandering, and the strength of the energetic interactions between steps. The value of $c_{z}$ can be roughly estimated from eq. (5) by assuming that the asymptotic form is valid down to $z=1$. This assumption has been shown to be good for SOS models of unstepped surfaces, for example [10]. Thus we estimate

$c_{z} \approx \exp \left[\left\langle(u(1,0)-u(0,0))^{2}\right\rangle \sin ^{2} \phi / 4 X\right]$,

i.e., $c_{z}$ depends exponentially on the mean square deviation of the terrace width about its mean value. This number can be straightforwardly computed exactly at low temperatures by, for example, applying the free fermion approximation to the TSK model [11]. One finds that $\langle(u(1,0)-$ $\left.u(0,0))^{2}\right\rangle \approx 14.6$ for a mean step separation of $l=8$ (and $\approx 0.18(l+1)^{2}$ in the limit of large $l$ ) compared to the value of 16.5 found in the fit in fig. 4. The value of $c_{y}$ governs the shapes of the beam profiles perpendicular to the direction of splitting (see below). For steps which do not have energetic intractions with each other, one expects [7,11]:

$c_{y} \propto(l / b)^{2} \propto l^{2} \exp (\epsilon / k T)$,

where $b$ is the root-mean-square size of each kink perpendicular to the step edge. Notice $c_{y}$ depends sensitively on the amount of step wandering and hence temperature (while $c_{z}$ does not, at least for TSK systems with no energetic interactions between steps [11]). By measuring the temperature dependence of $c_{y}$ one can estimate kink energies, as described in a forthcoming publication [11]. The relationship between step wandering and the form of diffracted profiles transverse to the splitting direction has been considered in ref. [12] for the case of energetically interacting steps.

An equation useful for analyzing heam profiles can now be derived by using eq. (5) in eq. (4) to give [13]:

$$
\begin{aligned}
S(\boldsymbol{q})= & I_{0} \sum_{n, m}\left[\left(\frac{q_{x} l+q_{z}-2 \pi n}{c_{z}}\right)^{2}\right. \\
& \left.+\left(\frac{q_{y}-2 \pi m}{c_{y}}\right)^{2}\right]^{-(2-\eta) / 2},
\end{aligned}
$$

with

$\eta=2 X q_{x}^{2} / \sin ^{2} \phi$, and

$$
I_{0}=2 \pi^{3 / 2} \frac{L_{z} L_{y}\left(1-\cos \left(q_{z}\right)\right)}{c_{z} c_{y}\left(1-\cos \left(q_{x}\right)\right)} \frac{\Gamma(2-\eta)}{\Gamma\left(\frac{\eta}{2}\right) \Gamma\left(\frac{3}{2}-\frac{1}{2} \eta\right)} .
$$

These equations can be used to evaluate beam profiles parallel to the direction of splitting (i.e., where $q_{y}$ is a constant) without the need for the parameter $c_{y}$. The primary information obtained from such analysis concerns the range of the step-step correlations, as gauged in terms of the value of $X$ (cf. eq. (5)). Eq. (9) is only valid when $\eta<2 ; \eta$ goes to zero in the limit of ordered steps. As expected for vicinal surfaces, the structure factor has the form characteristic of rough surfaces: power law divergences near the reciprocal lattice rods of the perfectly ordered surface. The full widths at half maximum of these peaks are zero: experimentally the widths will only be limited by instrumental resolution (or surface imperfections!). The value of $\eta$ determines how sharp the diffracted peaks are. The peaks are especially difficult to distinguish from $\delta$-functions when $\eta$ is small. In the limit of small angles $\left(l^{-1}=\tan \phi \approx\right.$ $\sin \phi$ ) one expects that $\eta$ will be approximately $\frac{1}{2}$ for the TSK model at out-of-phase conditions: $q_{x} \approx \pi / l$, so that $\eta \approx 2 X_{v} \pi^{2} \approx \frac{1}{2}$. This value should be universal in the sense that it is independent of such details as kink geometry and step height.

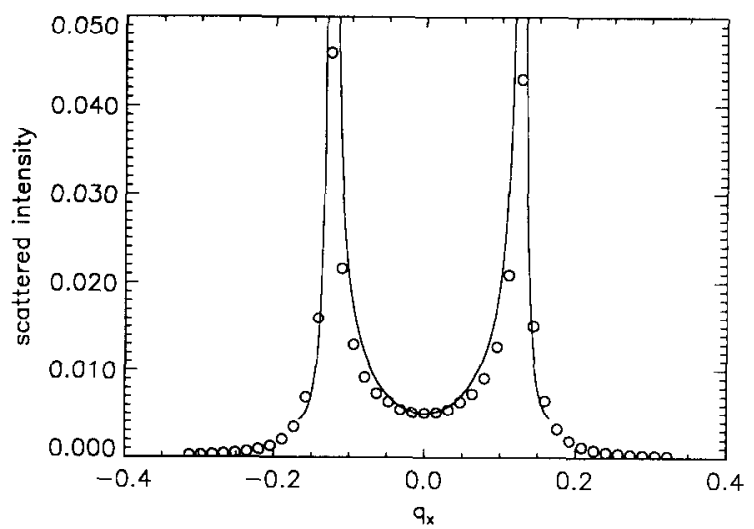

Fig. 5. Comparison between Monte Carlo calculation of the diffracted profile for the TSK model at $k_{\mathrm{B}} T=0.8 \epsilon$ (circles) and the asymptotic theory of eq. (9) with $c_{z}=5.0$ and $X=0.040$ (solid line) 


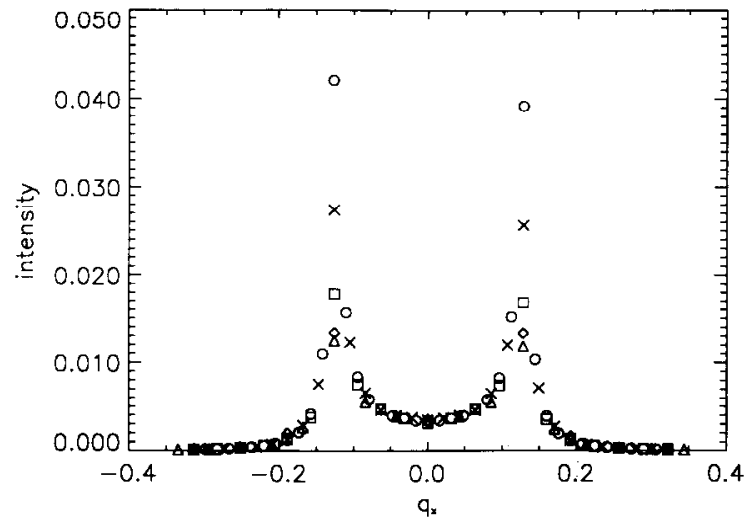

Fig. 6. Finite-size effects on the scattered intensity for the TSK model at $k_{\mathrm{B}} T=0.8 \mathrm{\epsilon}$. The circles correspond to lattices size $128 \times 128$, crosses to $96 \times 96$, squares to $64 \times 64$, diamonds to

$48 \times 48$, and triangles to $32 \times 32 . q_{x}$ is in units of $\pi / a$.

However (see section 7), it will depend on the range of step-step interactions.

Fig. 5 shows that there is good agreement between the form of $S(q)$ predicted in eqs. (9)-(11) and the Monte Carlo results for the TSK model at $k_{\mathrm{B}} T=0.8 \epsilon$, using the values of $c_{z}$ and $X$ found from fig. 4 . The value of $c_{y}$, which just determines the overall scale of the predicted curve parallel to the splitting direction, was chosen to give the best fit to the Monte Carlo data.

The computed peak intensity of the split beams does not diverge in fig. 5 because of finite-size effects. As the simulated system gets larger, the intensity of the split beams gets larger. This effect is shown in fig. 6, where the diffracted intensity for various $L=L_{x}=L_{y}$ is shown for the TSK model at $k_{\mathrm{B}} T=0.8 \epsilon$. The intensity in these plots is normalized per unit area. The intensity away from $q_{x}= \pm \pi / l$ converges quickly, while the intensity at $q_{x}= \pm \pi / l$ increases with $L$. If the structure factor became a $\delta$-function in the infinite system limit, one would expect this intensity to diverge like $L^{2}$. For a power-law diffraction peak as given by eq. (9), finite-size scaling theory [14] predicts a divergence like $L^{2-\eta_{0}}$, where $\eta_{0}=$ $2 X \pi^{2}\left(l^{2}+1\right) / l^{2}$ is the value of $\eta$ given by eq. (10), evaluated at the values of $q_{x}$ at the out-ofphase condition. Analysis of the intensities at $q_{x}$ $= \pm \pi / l$ from the data in fig. 6 yields $\eta_{0} \approx 0.8 \pm$ 0.1 , consistent with the value of $X$ found in fig. 4 .
In real experiments, this divergence will be masked by instrumental resolution. Diffraction instruments with finite spatial resolution will yield diffracted profiles without the singularities of eq. (9). However, increasing the instrumental resolution will cause the (normalized) peak intensities to increase in manner analogous to the finite-size increase of fig. 6: the peak intensities should diverge like $L_{\mathrm{I}}^{2-\eta_{0}}$ [15], where $L_{\mathrm{I}}$ is the "transfer width" of the instrument (compared with the $L_{1}^{2}$ divergence expected for a $\delta$-function peak). We again emphasize that one-dimensional models with short-range interactions do not have these divergences.

The previous discussion presents the form of the diffraction profiles for the equilibrium step structure with the largest amount of local disorder: non-interacting steps. We also mention the two limiting cases of very strong attractive or repulsive interactions. Attractive interactions can cause steps to coalesce to form a faceted surface. Repulsive interactions will stabilize the vicinal orientations and increase the step order, thus decreasing $X$ and sharpening the profiles. In the limit of very strong repulsions, $X$ becomes zero and the vicinal surface corresponds to a facet. Such a surface, containing an ordered staircase step structure, would also have sharp, albeit weak, diffraction peaks near $q_{x} \approx \pm 3 \pi / l, \pm 5 \pi / l, \ldots$ as shown in fig. $3 \mathrm{~b}$. These peaks are much more sensitive to disorder than the $q_{x}= \pm \pi / l$ peaks discussed above. This sensitivity can be clearly seen from eq. (10): the values of $\eta$ at these positions are very large at out-of-phase conditions: $\eta=\frac{9}{2}, \frac{25}{2}, \ldots$. When $\eta$ is larger than 2 , analysis of eq. (4) reveals that there are no divergences in $S(q)$. Correspondingly, our simulations show very small intensities at the higher-order diffraction conditions. Observations of sharp diffraction features at these conditions are thus grossly inconsistent with the type of thermal disorder anticipated on the bais of models of surfaces containing weakly interacting steps. As an application of this idea, the sharp higher order diffraction beams visible on $\mathrm{Ge}(111)$ misoriented by $10^{\circ}$ towards the [1 12 ] direction, would suggest that fig. 1 would hardly be an accurate description, despite the relatively small angle of misorientation [16]. 

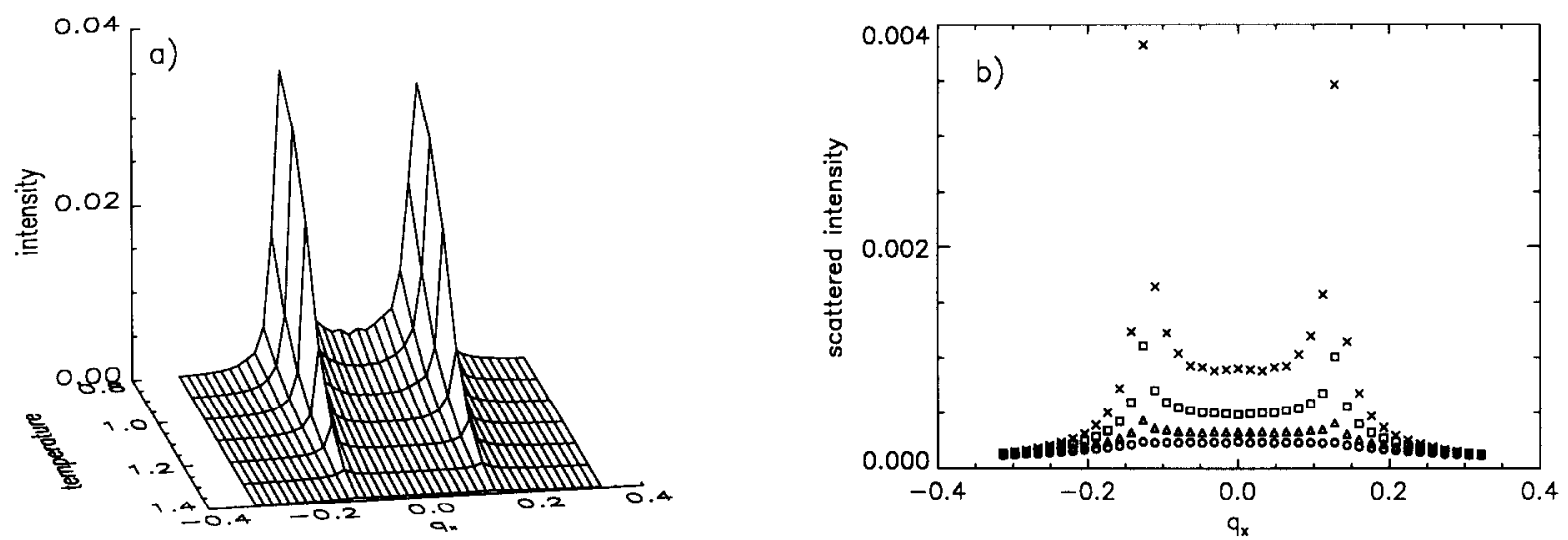

Fig. 7. SOS model diffraction profiles at an out-of-phase condition as a function of temperature. (a) A surface plot showing the large decrease in intensity near the roughening temperature of an infinitely large terrace $\left(k_{\mathrm{B}} T_{\mathrm{R}} \approx 1.2 \epsilon\right)$. (b) Several high temperature profiles are coplotted. Crosses correspond to $k_{\mathrm{B}} T=1.2 \epsilon$, squares to $k_{\mathrm{B}} T=1.3 \epsilon$, triangles to $k_{\mathrm{B}} T=1.4 \epsilon$, and circles to $k_{\mathrm{B}} T=1.5 \epsilon$, respectively. The double-peaked nature of the diffracted profiles does not disappear until above $k_{\mathrm{B}} T_{\mathrm{R}}$.
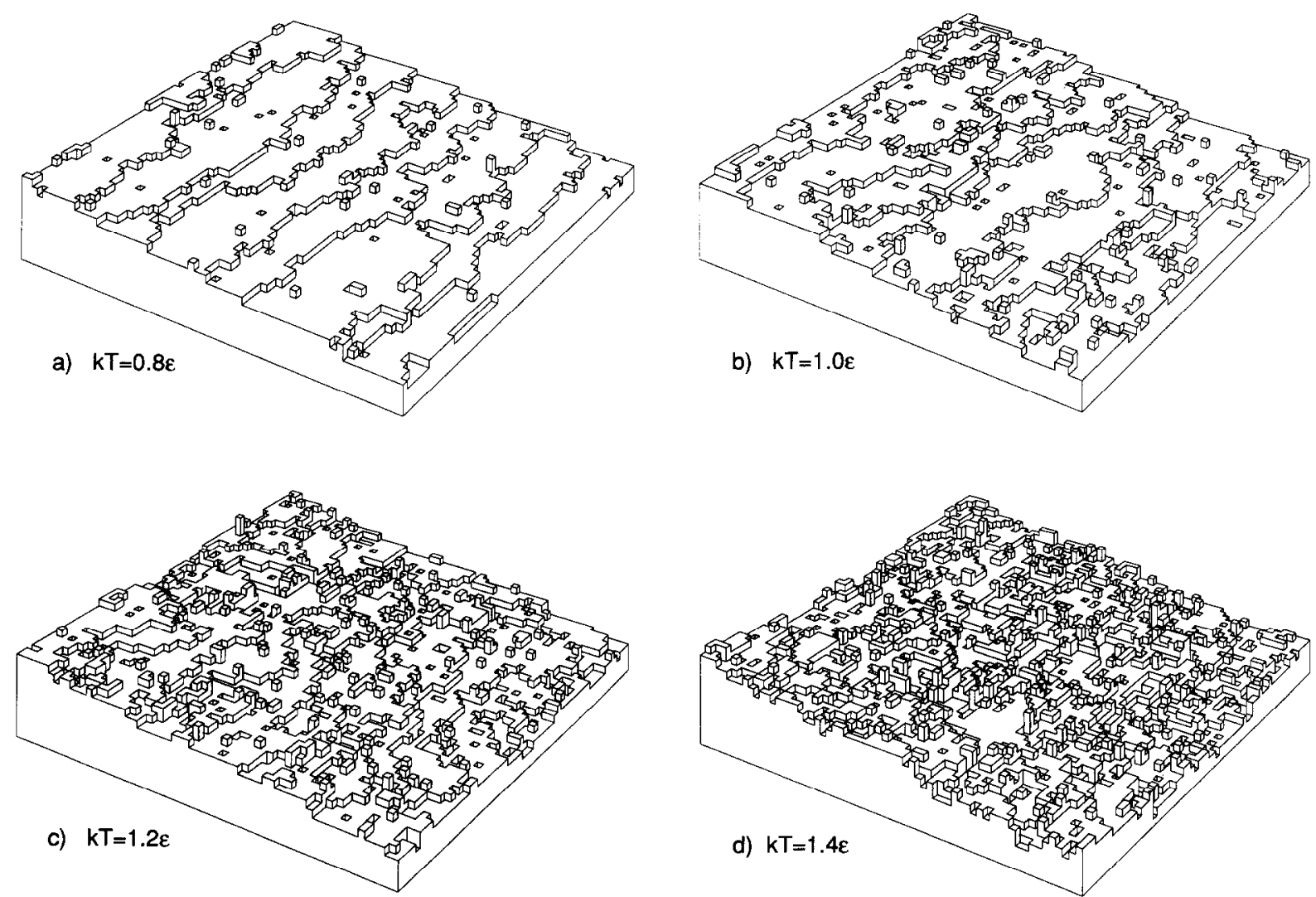

Fig. 8. SOS model surface configurations as a function of temperature for the same range of temperatures as in fig. 7: (a) $k_{\mathrm{B}} T=0.8 \epsilon$, (b) $k_{\mathrm{B}} T=1.0 \epsilon$, (c) $k_{\mathrm{B}} T=1.2 \epsilon$, (d) $k_{\mathrm{B}} T=1.4 \epsilon$. For clarity only surfaces of dimension $64 \times 64$ are shown, compared to the $128 \times 128$ surfaces used in computing the diffracted profiles. 


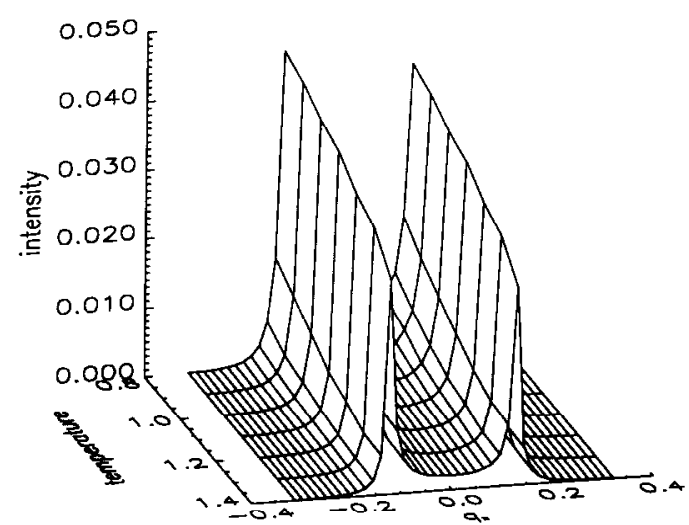

Fig. 9. TSK model diffraction profiles at an out-of-phase condition for the same range of temperatures as the SOS model given in fig. 7. The changes are much smaller because the lack of terrace excitations reduces the decay of height-height correlations. $q_{x}$ is in units of $\pi / a$.

\section{Temperature dependence of the diffracted profiles}

From eqs. (9) and (10), the smaller the value of $X$ the sharper the diffraction features at out-ofphase conditions. As one increases the number of terrace excitations in the SOS model by increasing temperature, one expects the value of $X$ to increase [17]. The effect of this increase is illustrated in fig. 7 , which shows a sequence of diffraction profiles at an out-of-phase condition. As the temperature is raised, the intensity of the peak splittings gradually becomes smaller, but still easily visible. On the other hand, fig. 8, which contains corresponding sample SOS surface configurations, shows clearly that considerable surface disorder is present under conditions showing split diffraction features. Even near the roughening temperature of the (100) facet in this model $\left(k_{\mathrm{B}} T_{\mathrm{R}} \approx 1.24 \epsilon[6]\right)$ where steps are barely discernible (or even definable [3]) there is still a well-defined splitting. Only above the roughening temperature of the (100) facet does the splitting vanish.

Fig. 9 shows the peak intensity of the split beams at an out-of-phase condition as a function of temperature for the TSK model. The splittings in the profiles for the TSK model persist to large temperatures, and have a shape which is largely temperature-independent. This behavior is con- sistent with the expected universality of $X$ in the limit of small misorientation angles below the roughening temperature of the $\phi=0$ surface: since the terraces do not roughen in the TSK model, $X$ should not vary much for small but finite angles.

\section{Energy dependence of the diffracted profiles}

To demonstrate that a stepped surface is in thermal equilibrium, one must show that the diffraction profiles have the power-law divergences of eq. (9). To determine the nature of the step interactions, it is necessary to determine the strength of the step correlations by measuring the value of $\eta$. In principle, one could extract $\eta$ and thus $X$ from experimental diffraction profiles by directly plotting $\log S$ versus $\log \left(q_{x} l+q_{z}-2 \pi n\right)$. The strong dependence of $\eta$ on $q_{x}$ in eq. (10) makes this procedure ineffective for experimentally accessible ranges of $q_{x}$ : the $\log -\log$ plots are far from linear over the range of $q_{x}$ shown in fig. 3. A better approach [11], sometimes used in studies of surface roughening, is to analyze profiles at constant $q_{x}$ [18]. Here we describe an alternative approach based on the analysis of the peak intensities as the incident energy, i.e. $q_{z}$, is varied.

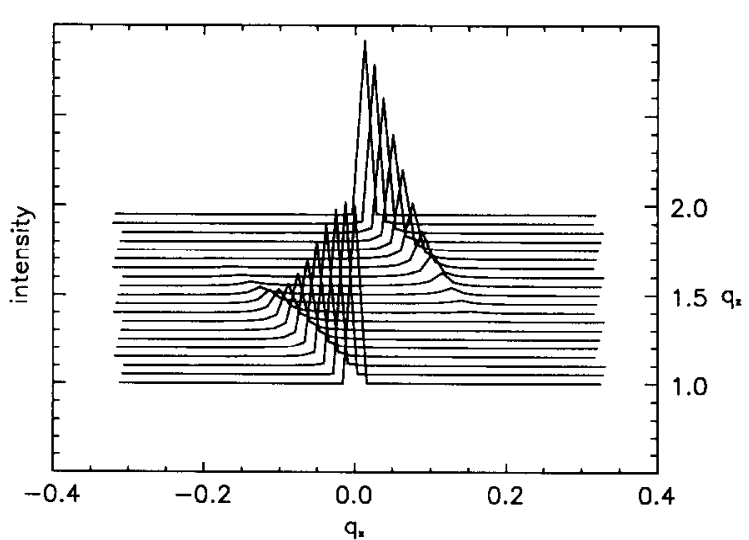

Fig. 10. The dependence of diffracted intensity of the TSK model on incident momentum at $k_{\mathrm{B}} T=0.8 \mathrm{\epsilon} . q_{x}$ and $q_{z}$ are in units of $\pi / a$. The bascline of each of the curves is displaced by $\Delta q_{z}=0.05 \pi / a$ as shown on the right-hand axis. Intensities were calculated at intervals of $\Delta q_{x} \sim \pi / L_{x}$; thus, the discontinuities in the curves are a computational artifact. Notice the sharp drop in intensity near the out-of-phase condition. 


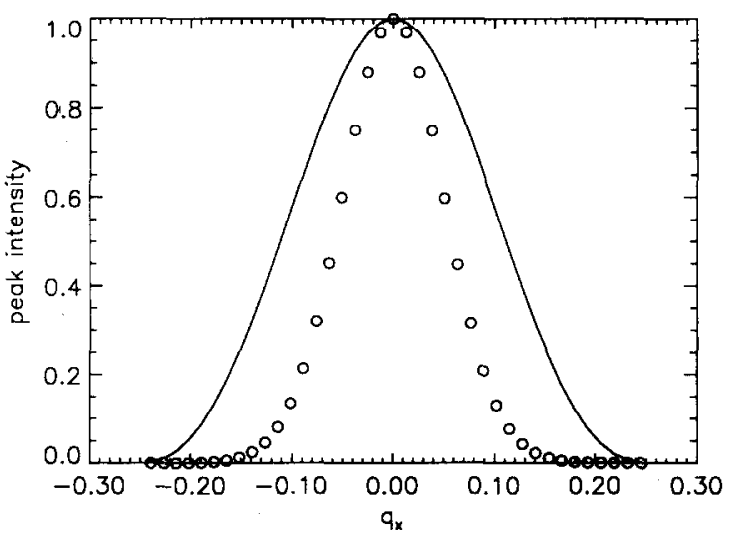

Fig. 11. The diffracted intensity at $q_{x} l+q_{z}=2 \pi$ for the TSK model at $k T=0.8 \epsilon$ (circles) as a function of the $q_{x}$ of the peak position, compared to the variation for a perfectly ordered staircase (solid line). $q_{x}$ is in units of $\pi / a$.

Fig. 10 shows the dependence of the scattered intensity near $q_{x}=0$ as the incident energy is varied from an in-phase condition through an out-of-phase condition to the next in-phase condition. The behavior of these profiles reveals several features predicted by eq. (9). First, the intensity of the beams diminishes rapidly as the position of the peak approaches $\pi / l$, its value at the first out-of-phase condition. (If the surface were perfectly ordered, one would likewise expect a decay with increasing $q_{x}$. However, this decay is significantly slower than the behavior we observe, as indicated by the solid line in fig. 11.) Eq. (9) predicts that there will be no singularity in the diffracted intensity when $\eta=2$, i.e., when $q_{x}=$ $\sin \phi / X^{1 / 2}$ (cf. eq. (10)). As shown in fig. 11, our Monte Carlo simulations show no intensity in the split beam when $\left|q_{x}\right|>1.4 \pi / l=0.175 \pi / a$. If one interprets the disappearance of the peak as indicating $\eta=2$, this observation yields the estimate of $X \approx 0.05$. This $X$ is consistent with the value of $X$ deduced above from direct observation of the height-height correlation function, and also from finite size scaling. Thus we have a straightforward way of estimating the value of $X$, or at least for putting an upper limit on its value, without performing fits to diffracted profiles. This method might be difficult in LEED because multiple scattering complicates interpretation of the dependence of scattered intensities on incident electron energy.

\section{Discussion}

In this paper we have shown that even in the absence of energetic step-step interactions, steps in thermal equilibrium give rise to sharp diffraction features, the full widths at half maximum of the beams at out-of-phase conditions being limited only by instrumental resolution: Ultimately the sharpness of the beams is determined by the longrange correlations in surface height (through eq. (4)). In a two-dimensional description, the divergences one expects for these correlations (because of step wandering) are relatively small (i.e. logarithmic, cf. eq. (5)). One thus must be careful in trying to relate beam broadening to short-range quantities such as terrace width distributions, as is done, for example, in refs. [2] and [19]. In particular, many different possible height-height correlations can arise from the same distribution of terrace widths. For example, in the one-dimensional model, the same distribution of terrace widths which yields the unsplit diffraction profile in fig. $3 \mathrm{a}$, could equally yield $\delta$-functions if one imposed an additional constraint restricting surface height fluctuations, i.e., by hypothesizing correlations between neighboring terrace widths. Such a constraint could be supplied by restricted surface diffusion.

When interpreting experimental diffraction profiles in terms of the types of statistical mechanical calculations presented here, an important caveat concerns the question of equilibrium. Surfaces are usually prepared under conditions which are very far from equilibrium. For example, stepped Si surfaces, which have been the subject of intense scrutiny lately [20], are usually cleaned by heating to temperatures at which a large amount of sublimation occurs. High-energy reflection electron microscopy has shown clearly that step structure at these temperatures can be dominated by kinetic effects [21]. It is far from obvious that an equilibrium configuration will occur when one cools, especially when the steps are widely spaced. Whether or not the surface is in equilibrium will depend on surface diffusion, which is generally poorly characterized. The question of whether kinetically determined surfaces would give rise to sharper or more diffuse diffraction features than 
the ones presented here is also difficult to answer a priori: there are plausible kinetic mechanisms which can cause surfaces to be rougher $[22,23]$ or smoother [24] than they would be in equilibrium. However, our calculations do show that even when steps do not interact with each other energetically over large distances, sharp diffraction features are generally expected from stepped surfaces in equilibrium.

Interactions between steps will undoubtedly affect the shape of the diffracted profiles. As an extreme case, long-range repulsive step-step interactions can stabilize high-index facets, leading to peaks with $\delta$-function components in the diffracted intensity at out-of-phase conditions at low temperatures. One might anticipate that the roughening temperature induced by these interactions would be low for small angles of misorientation, however. Attractive interactions can increase $X$ [9], making the surface rougher than the noninteracting case, by causing incipient faceting of the surface [25].

\section{Acknowledgements}

This work was supported by the NSF under grant DMR-8802986. Computer time was supplied by the Pittsburgh Supercomputer Center.

\section{References}

[1] M. Henzler, Surf. Sci. 22 (1970) 12.

[2] P.R. Pukite, C.S. Lent and P.I. Cohen, Surf. Sci. 161 (1985) 39, and references thcrein; see also, more recently, L.H. Zhao and M. Henzler, Appl. Phys. A 50 (1990) 595.

[3] For recent reviews, see: H. van Beijeren and I. Nolden, in: Structure and Dynamics of Surfaces II, Eds. W. Schommers and P. von Blanckenhagen (Springer, Berlin, 1987) p. 259;

M. Wortis, in: Chemistry and Physics of Solid Surfaces VII, Eds. R. Vanselow and R. Howe (Springer. Berlin, 1988) p. 367.

[4] This observation was perhaps first made by P.-G. de Gennes, J., Chem. Phys. 48 (1968) 2257, in the context of a simple model of flexible chains with only steric constraints;

see also J. Villain, in: Ordering in Strongly Fluctuating Condensed Matter Systems, Ed. T. Riste (Plenum, New York, 1980) p. 235.

[5] W. Selke and A.M. Szpilka, Z. Phys. B 62 (1986) 381.

[6] See, for example, J.D. Weeks, in: Ordering in Strongly Fluctuating Condensed Matter Systems, Ed. T. Riste (Plenum, New York, 1980) p. 293.

[7] M.E. Fisher, J. Stat. Phys. 34 (1984) 667.

[8] J. Villain, D.R. Grempel and J. Lapujoulade, J. Phys. F 15 (1985) 809.

[9] W.F. Saam, Phys. Rev. Lett. 62 (1989) 2636.

[10] W.J. Shugard, J.D. Weeks and G.H. Gilmer, Phys. Rev. Lett. 41 (1979) 1399; J. Adler, Phys. Rev. B 36 (1987) 2473.

[11] N.C. Bartelt, J.L. Goldberg and T.L. Einstein, in preparation.

[12] R. Kariotis, B.S. Swartzentruber and M.G. Lagally, J. Appl. Phys. 67 (1990) 2848.

[13] We differ from the results of ref. [8] by a factor of $2 \pi^{3 / 2}$.

[14] P. Nightingale, J. Appl. Phys. 53 (1982) 7927.

[15] N.C. Bartelt, T.L. Einstein and L.D. Roelofs, Phys. Rev. B 32 (1985) 2993.

[16] T.M. Jung, R.J. Phaneuf and E.D. Williams, in preparation.

[17] When the surface orientation corresponding to the terraces becomes "rough," $X$ is expected to have the universal value $1 / 2 \pi^{2}$, i.e. twice as large as the universal value predicted for vicinal surfaces in the limit of small misorientation angles [9], and increases above the roughening temperature [3].

[18] I.K. Robinson, E.H. Conrad and D.S. Reed, J. Phys. (Paris) 51 (1990) 103.

[19] D. Saloner, J.A. Martin, M.C. Tringides, D.E. Savage, C.E. Aumann and M.G. Lagally, J. Appl. Phys. 61 (1987) 2884.

[20] R.J. Phaneuf and E.D. Williams, Phys. Rev. Lett. 58 (1987) 2563; Phys. Rev. B 41 (1990) 2991;

R.J. Phaneuf, E.D. Williams and N.C. Bartelt, Phys. Rev. B 38 (1988) 1984;

B.S. Swartzentruber, Y.-W. Mo, M.B. Webb and M.G. Lagally, J. Vac. Sci. Technol. A 7 (1989) 2901;

J.E. Griffith and G.P. Kochanski, Crit. Rev. in Solid State and Mater. Sci. 16 (1990) 255.

[21] A.V. Latyshev, A.L. Aseev, A.B. Krasilnikov and S.I. Stenin, Surf. Sci. 213 (1989) 157.

[22] M. Kardar, G. Parisi and Yi-Cheng Zhang, Phys. Rev. Lett. 56 (1986) 889.

[23] G.S. Bales and A. Zangwill, Phys. Rev. B 41 (1990) 5500.

[24] R.L. Schwoebel and E.J. Shipsey, J. Appl. Phys. 37 (1966) 3682 .

[25] C. Jayaprakash, C. Rottman and W.F. Saam, Phys. Rev. B 30 (1984) 6549. 